\title{
DIAGNÓSTICO Y TRATAMIENTO DE LA CORIOAMNIONITIS CLÍNICA
}

\section{Diagnosis and treatment of clinic chorioamnionitis}

Franklin J. Espitia-De La Hoz, M.D.*

Recibido: abril 1/08 - Aceptado: agosto 19/08

\section{RESUMEN}

Objetivo: el objetivo del presente trabajo es revisar algunos aspectos del diagnóstico y tratamiento de la corioamnionitis.

Materiales y métodos: se realizó una búsqueda bibliográfica en diferentes bases de datos electrónicas para identificar literatura relevante: Pubmed, Ovid, Elsevier, Interscience, Medline, EBSCO, Scopus, SciELO (1980 al 2008) y Cochrane Pregnancy and Childbirth Group (marzo 31 del 2008) y libros de texto impresos, tratando de encontrar la mejor evidencia respecto al diagnóstico y tratamiento de la corioamnionitis.

Resultados: la corioamnionitis es un síndrome que puede afectar, tanto a la madre como al feto, y que debido a sus potenciales repercusiones en el binomio madre-feto, requiere de un diagnóstico y tratamiento oportuno.

Conclusión: la sospecha clínica de corioamnionitis es una condición catastrófica del bienestar fetal, que puede desencadenar un parto pretérmino o que potencialmente puede obligar a la inducción del parto con un feto prematuro.

Palabras clave: corioamnionitis, infección intraamniótica, diagnóstico y terapia antibiótica.

\section{SUMMARY}

Objective: the present work was aimed at reviewing some aspects of chorioamnionitis diagnosis and treatment.

* Residente de ginecología y obstetricia, Universidad Militar Nueva Granada. Bogotá, Colombia. Correo electrónico: espitiafranklin@hotmail.com
Materials and methods: a search was made of Pubmed, Ovid, Elsevier, Interscience, Medline, EBSCO, Scopus, SciELO (1980 to 2008) and Cochrane Pregnancy and Childbirth Group (March 31st 2008) electronic databases and printed text books to identify literature giving the best evidence regarding chorioamnionitis diagnosis and treatment.

Results: chorioamnionitis is a syndrome which can affect both the mother and the foetus, requiring opportune diagnose and treatment due to its potential repercussions on mother-foetus binomial distribution.

Conclusion: clinical suspicion of chorioamnionitis is a catastrophic condition of the foetus which can trigger preterm childbirth or may force induction of childbirth thereby leading to a premature foetus.

Key words: chorioamnionitis, intra-amniotic infection, diagnosis, antibiotic therapy.

\section{INTRODUCCIÓN}

La corioamnionitis es una infección del líquido amniótico y las membranas que lo contienen; también se denomina infección intraamniótica o amnionitis y puede ir acompañada de una ruptura prematura de membranas o con el saco amniótico completo. ${ }^{1}$ Se asocia a una mayor morbimortalidad materna y neonatal, especialmente en recién nacidos pretérmino. $^{2,3}$

La corioamnionitis clínica complica entre el 2 y el 11\% de todos los embarazos y en aproximadamente el 5\% de los casos el feto está infectado; ${ }^{4}$ 
siendo mucho más común en los partos prematuros. ${ }^{5,6}$

La incidencia de corioamnionitis histológica subclínica es mucho más común en embarazos pretérmino: $40 \%$ entre 24 y 28 semanas, 30\% entre 28 y 32 semanas, 20\% entre 30 y 36 semanas, y 10\% en embarazos mayores de 37 semanas. ${ }^{7,8}$

En la actualidad, existe abundante evidencia biomédica, clínica y epidemiológica que permite establecer una relación causa-efecto entre la corioamnionitis y el desencadenamiento del parto prematuro; y ya no se discute la existencia de especificidad, consistencia y temporalidad de asociación entre esta infección con el parto pretérmino y/o la ruptura prematura de membranas. ${ }^{9}$

Inicialmente la corioamnionitis fue acuñada como un término anatomopatológico que se refería a la infiltración leucocitaria de la placenta. Posteriormente, este mismo término ha sido utilizado para definir la infección clínicamente evidente de la gestante, el feto, placenta y membranas. Sin embargo, ambos cuadros no son equivalentes ni se presentan a menudo de forma conjunta. ${ }^{1,3}$

El objetivo de este documento es revisar los aspectos diagnósticos y de tratamiento de esta entidad.

\section{MATERIALES Y MÉTODOS}

Con las palabras claves chorioamnionitis or amnionitis and diagnosis and reatment, se realizó una búsqueda bibliográfica en diferentes bases de datos electrónicas para identificar literatura relevante: Pubmed, Ovid, Elsevier, Interscience, Medline, EBSCO, Scopus, SciELO (1980 al 2008) y Cochrane Pregnancy and Childbirth Group (marzo 31 del 2008) y libros de texto impresos.

\section{DIAGNÓSTICO}

El diagnóstico de la corioamnionitis es básicamente clínico y es una infección que se debe descartar en toda embarazada que presente fiebre sin foco aparente, sobre todo si se sospecha o se ha confirmado una rotura de membranas. ${ }^{9}$
En el $80 \%$ de los casos el curso de la corioamnionitis es subclínico, por lo que el diagnóstico se basará en la identificación de las complicaciones. Con frecuencia, no se manifiesta hasta después del parto, cuando la madre desarrolla fiebre en el postparto precoz o irritabilidad uterina o el feto presenta mal olor o signos de infección. ${ }^{10}$ Otro signo de sospecha en las gestaciones pretérmino, es la presencia de amenaza de parto prematuro refractaria al manejo.

El diagnóstico de corioamnionitis clínica se realiza mediante los siguientes datos clínicos, que fueron establecidos por Gibbs y cols: ${ }^{5}$

Temperatura axilar igual o mayor a $38^{\circ} \mathrm{C}$, acompañada de dos o más de los siguientes signos (algunos autores han abogado por un umbral más bajo de $\left.37,8^{\circ} \mathrm{C}\right):^{11}$

a) Sensibilidad uterina anormal.

b) Líquido amniótico purulento o de mal olor.

c) Taquicardia materna mayor de 100 latidos por minuto.

d) Taquicardia fetal mayor de 160 latidos por minuto.

e) Leucocitosis $>15.000 / \mathrm{mm}^{3}$.

f) Aumento de la contractibilidad uterina.

g) Dolor pélvico al movimiento.

- Tinción de Gram con gérmenes (recordar que Mycoplasma hominis y Ureaplasma urealyticum, Chlamydiae trachomatis no se tiñen con esta técnica). La presencia de cualquier bacteria y la presencia de leucocitos es sugestiva de infección.

- Recuento de glóbulos blancos >50 células por $\mathrm{mm}^{3}$.

- Glucosa $<15$ mg/dl.

- Cultivos corriente, anaerobio, micoplasma/ureaplasma (medio especial para Chlamydiae).

- Pueden solicitarse estudios de madurez pulmonar después de las 30-32 semanas.

Estos criterios tienen sensibilidad que oscila entre el 24 y 81\%, especificidad entre el 60 y 99\%, valor predictivo positivo entre 42 y $91 \%$ y valor predictivo negativo entre 68 y $86 \% .^{12}$ 


\section{Exámenes de laboratorio}

La inespecificidad de la mayoría de los laboratorios obliga a realizar el diagnóstico a través de la conjugación de muchos de éstos.

- Cultivo del líquido amniótico: tiene como desventaja el tiempo prolongado para la obtención de resultados, haciéndolo poco útil clínicamente; además que el cultivo no identifica infecciones localizadas en la decidua y corion, que podrían ocurrir sin la invasión bacteriana hacia cavidad amniótica. ${ }^{13,14}$

- Proteína C reactiva aumentada: suele aumentarse 2 ó 3 días antes de la sintomatología clínica, ${ }^{15}$ es producto de la reacción hepática de fase aguda a la infección, en respuesta a la síntesis de IL-6 sintetizada durante el curso de la infección.

- La tinción de Gram: se realiza en líquido amniótico no centrifugado, para detectar la presencia de bacterias y leucocitos; sin embargo, se necesitan aproximadamente 10 microorganismos por $\mathrm{mL}$ para que sea positiva y no identifica micoplasmas, altamente reconocidos como grupo importante de causa de corioamnionitis. ${ }^{9}$

- El nivel bajo de glucosa en líquido amniótico con valores inferiores a $15 \mathrm{mg} / \mathrm{dl}$ es sugestivo, ${ }^{16}$ disminución probablemente secundaria al metabolismo, tanto bacteriano como de los neutrófilos.

- Leucocitosis materna mayor a 15.000 con desviación a la izquierda y con neutrófilos en banda superiores a 5\%, aunque presenta bajos niveles de sensibilidad y de valor predictivo positivo, lo que lo hace inadecuado para su uso en la práctica clínica por ser una prueba inespecífica, ya que es un estándar clínico de infección sistémica grave. ${ }^{17}$ Algunos autores afirman que cuando el recuento es superior a 12.000 la sensibilidad es del $67 \%$ y el valor predictivo positivo del $82 \%$, para el diagnóstico de infección del LA. ${ }^{18}$

- Hemocultivo positivo: también tiene como desventaja el tiempo prolongado para la obtención de resultados, siendo poco útil clínicamente, ${ }^{13}$ además que sería diagnóstico de bacteriemia como eventual complicación tardía.
- Perfil biofísico: se ha reportado que una puntuación menor o igual a 7, cuya realización haya sido en las 24 horas previas a la interrupción del embarazo, es un buen factor predictivo de sepsis neonatal, y entre más variables estén comprometidas, mayor correlación existe con la infección fetal. ${ }^{19}$

- Los niveles de deshidrogenasa láctica (LDH) en líquido amniótico se han visto elevados en presencia de corioamnionitis y los conceptos actuales la señalan como un predictor altamente específico y precoz para infección intraamniótica. El valor predictor de corioamnionitis es $410 \mathrm{u} / \mathrm{L}$ en líquido amniótico. ${ }^{20}$

- Esterasa leucocitaria: es un producto de los leucocitos polimorfonucleares cuya actividad se incrementa en presencia de infección amniótica. Su positividad en el líquido amniótico tiene una sensibilidad del 91\%, especificidad del 84\%, y un valor predictivo positivo del $95 \%$ y negativo del $74 \% .^{21}$

En la actualidad están en estudio las pruebas de diagnóstico rápido de infección. Por ejemplo, los niveles elevados de interleucina 6 en el líquido amniótico, que predicen la corioamnionitis con un $100 \%$ de sensibilidad y un $83 \%$ de especificidad. Algunos autores han concluido que la interleucina 6 es el mejor marcador de la infección intraamniótica; ${ }^{16}$ otros por su parte, definen la corioamnionitis cuando los niveles de IL-6 en el líquido amniótico, están mayores de 2,6 ng/mL; $;^{22}$ y en recientes artículos se ha mencionado que los niveles elevados de interleucina 6 en secreciones vaginales tienen un valor predictivo significativo de infección intraamniótica. ${ }^{23}$

\section{TRATAMIENTO}

Cuando se realiza el diagnóstico de corioamnionitis, el consenso actual es la administración intraparto de los antibióticos; sin embargo, los resultados no apoyan ni refutan esto, aunque existe una tendencia hacia mejores resultados neonatales cuando los antibióticos se administran intraparto; pero definiti- 
vamente la corioamnionitis es mejor prevenirla que tratarla. ${ }^{24,25}$ Se debe tener en cuenta el cubrimiento de gérmenes que ya se sabe que se involucran con frecuencia en dicha infección. ${ }^{26}$

Las pacientes con infección intraamniótica generalmente no responden al tratamiento tocolítico y deben ser manejadas, preferentemente, con antibióticos endovenosos:

a) Si hay corioamnionitis clínica: terapia antibiótica endovenosa. Si la dinámica uterina se detiene o es escasa, inducir o conducir, respectivamente, para provocar el parto dentro de las siguientes 6-12 horas; la cesárea sólo estará indicada bajo indicaciones obstétricas. ${ }^{5,27}$

b) Si hay una infección intraamniótica subclínica en una paciente con contractilidad uterina, debe suspenderse la terapia tocolítica y agregar antibióticos. La gran mayoría de las pacientes evolucionará a fase activa en forma espontánea. Si la paciente no evoluciona hacia fase activa, las situaciones pueden ser:

En la paciente con corioamnionitis diagnosticada durante el trabajo, muchos autores han defendido el tratamiento antibiótico parenteral continuo, hasta que la paciente ha logrado permanecer sin fiebre y asintomática por un periodo no menor de 24 a 48 horas. ${ }^{28,29}$

En embarazo a término: inducir el trabajo de parto dentro las 6 primeras horas de haber hecho el diagnóstico. ${ }^{30}$ Tratar de realizar el menor número posible de tactos vaginales. En todas las situaciones y siempre que la situación obstétrica así lo permita, se procurará que la finalización del embarazo sea por la vía vaginal, con el objeto de minimizar el riesgo de infección abdominal. ${ }^{31}$

En embarazo de 34 a 36 semanas: proceder como si fuera a término, puesto que el riesgo de morbilidad y mortalidad neonatal por inmadurez pulmonar, después de cumplidas las 34 semanas de gestación, es muy bajo. ${ }^{30,32}$

En embarazo de 30 a 33 semanas: estimular madurez pulmonar fetal: $12 \mathrm{mg}$ betametasona intramuscular cada 24 horas por dos ocasiones, hasta completar 48 horas de la primera dosis o 24 horas de la segunda; ${ }^{33}$ instaurar la antibioticoterapia inmediatamente se haga el diagnóstico. La interrupción puede efectuarse de inmediato si se confirma la presencia de un pulmón maduro., ${ }^{9,34}$

Se podría manejar el embarazo en forma expectante, bajo tratamiento antibiótico y supervisión materna y fetal estricta, ya que la conducta clínica requiere un alto grado de individualización, debido a que no hay información suficiente para manejar estas pacientes en forma "estándar". ${ }^{34}$

En embarazo de 25 semanas y menor de 30 semanas: cada día que pasa incrementa la sobrevida y reduce la morbilidad neonatal en un $1-5 \%$, por lo que la ganancia en uno o dos días es mucho más significativa; siendo relevante las ganancias de más días. En el pronóstico juega un rol importante el volumen del líquido amniótico; el oligohidramnios severo da lugar a una hipoplasia pulmonar fetal o defectos musculoesqueléticos severos. ${ }^{9,34}$

En embarazo menor de 24 semanas: si el diagnóstico se realiza antes de las 24 semanas, el parto inmediato es el plan más eficiente en cuanto a la relación de morbi-mortalidad y costo-beneficio. ${ }^{9}$ Una vez que se haya producido el parto, mantener la antibioticoterapia mínimo 7 días durante el puerperio. $^{36}$

\section{1) Interrupción del embarazo por cesárea:}

En caso de existir una indicación formal de cesárea, ésta se debe realizar con los máximos cuidados de asepsia y debe tenerse presente lo siguiente:

a) La incidencia de infecciones es similar entre las vías de abordaje.

b) El abordaje extraperitoneal no tiene ventajas sobre el abordaje clásico.

c) Evitar el escurrimiento de líquido amniótico mediante el uso de compresas.

d) Evitar el uso indiscriminado de electrobisturí.

e) Realizar lavados prolijos en los tejidos que se puedan infectar.

f) Cambio de guantes para el cierre de la pared abdominal. 


\section{2) Antibioticoterapia:}

a) Penicilina cristalina 5 millones cada 6 horas $^{30}$ (alergia: eritromicina $0,5-1$ gramo cada 6-8 horas.) + gentamicina 3-5 mg/kg/día, una dosis diaria. ${ }^{29}$ Una dosis de carga de 5 millones de unidades intravenosas de penicilina y 2 millones de unidades intravenosas cada 4 horas o 2 gramos de ampicilina intravenosa cada 4 horas, son efectivas en un 90 a 95\% para reducir la colonización y la sepsis neonatal. ${ }^{37}$

b) Ampicilina 2 gramos cada 6 horas $^{9}+$ gentamicina 3-5 mg/kg/día, una dosis diaria.

c) Ampicilina sulbactam 2 gramos cada 8 horas $^{29}$ + clindamicina 600-900 mg cada 8 horas $^{29}$ (si la paciente requiere una operación cesárea, a fin de proporcionar una mayor cobertura microbiana contra anaerobios).

d) Ceftriaxona 1 gramo cada 12 horas + clindamicina 600-900 mg cada 8 horas. ${ }^{29}$

e) Metronidazol en dosis de carga de $15 \mathrm{mg} / \mathrm{kg}$, seguida de 7,5 mg/ $\mathrm{kg}^{38}$ (si la paciente requiere una operación cesárea, a fin de proporcionar una mayor cobertura microbiana contra anaerobios) + ampicilina sulbactam 2 gramos cada 8 horas. $^{39}$

Dado que el principal factor de riesgo conocido para infección intraamniótica por hongos es la asociación de embarazo a DIU o cerclaje, en pacientes con DIU adicionar fluconazol $400 \mathrm{mg}$ día endovenosos, ya que el 25\% de las pacientes tienen infección por hongos, predominando la Cándida. ${ }^{40,41}$

\section{AGRADECIMIENTOS}

Al Doctor Jaime Gallego Arbeláez, por su oportuna contribución en la revisión y análisis de este artículo, como también por sus sugerencias en el contenido del mismo.

\section{REFERENCIAS}

1. Hillier SL, Martius J, Krohn M, Kivian N, Holmes KK, Eschenbach DA. A case-control study of chorioamnionitis infection and histologic chorioamnionitis in prematurity. N Engl J Med 1988;319:972-8.
2. Armer TL, Duff P. Intraamniotic infection in patients with intact membranes and preterm labor. Obstet Gynecol Surv 1991;46:589-93.

3. Gibbs R, Romero R, HIller SL, Eschenbach DA, Sweet RL. A review of premature birth and subclinical infection. Am J obstet Gynecol 1992;166;1515-28.

4. Soper DE, Mayhall CG, Dalton HP. Risk factors for intraamniotic infection: a prospective epidemiologic study. Am J Obstet Gynecol 1989;161:562-8.

5. Gibbs RS, Castillo MS, Rodgers PJ. Management of acute chorioamnionitis. Am J Obstet Gynecol 1980;136:709-13.

6. Gibbs RS. Obstetric factors associated with infections of the fetus and newborn infant. En: Remington JS, Klein JO, eds. Infectious diseases of the fetus and newborn infant. Philadelphia: WB Saunders; 1995. p. 1241-63.

7. Romero R, Quintero R, Oyarzum E, Sabo V, Mazor M, et al. Intraamniotic infection and the onset of labor in preterm premature rupture of the membranes. Am J Obstet Gynecol 1988;159:661.

8. Parry S, Strauss JF 3rd. Premature rupture of the fetal membranes. N Engl J Med 1998;338:663-70.

9. Goldenberg R, Hauth JC, Andrews WW. Intrauterine infection and preterm delivery. N Eng J Med 2000;342:1500-7.

10. Romero R, Salafia C, Athanassiadis AP, Hanaoka S, Mazor M, Sepúlveda W, et al. The relationship between acute inflammatory lesions of the preterm placenta and amniotic fluid microbiology. Am J Obstet Gynecol 1992;166:1382-8.

11. Gibbs RS, Duff P. Progress in pathogenesis and management of clinical intraamniotic infection. Am J Obstet Gynecol 1991;164:1317-26.

12. Canavan TP, Simhan HN, Caritis S. An evidence-based approach to the evaluation and treatment of premature rupture of membranes: Part II. Obstet Gynecol Surv 2004;59:678-89.

13. Greig PC. The diagnosis of intrauterine infection in women with preterm premature rupture of the membranes (PPROM). Clin Obstet Gynecol 1998;41:849-63.

14. Blackwell SC, Berry SM. Role of amniocentesis for the diagnosis of subclinical intramniotic infection in preterm rupture of the membranes. Curr Opin Obstet Gynecol 1999;11:541-7. 
15. Nowak M, Oszukowski P, Szpakowski M, Malinowski A, Maciolek-Blewniewska G. [Intrauterine infections. The role of C-reactive protein, white blood cell count and erythrocyte sedimentation rate in pregnant women in the detection of intrauterine infection after preliminary rupture of membranes]. Ginekol Pol 1998;69:615-22.

16. Romero R, Yoon BH, Mazor M, Gómez R, González $\mathrm{R}$, Diamond MP, et al. A comparative study of the diagnostic performance of amniotic fluid glucose, white blood cell count, interleukin-6, and gram stain in the detection of microbial invasion in patients with preterm premature rupture of membranes. Am J Obstet Gynecol 1993;169:839-51.

17. Yoon BH, Jun JK, Park KH, Syn HC, Gómez R, Romero R. Serum C-reactive protein, white blood cell count, and amniotic fluid white blood cell count in women with preterm premature rupture of membranes. Obstet Gynecol 1996;88:1034-40.

18. Hoskins IA, Johnson TRB, Winkel CA. Leukocyte esterase activity in human amniotic fluid for the rapid detection of chorioamnionitis. Am J Obstet Gynecol 1987;157:730-732.

19. Vintzileos MA, Campbell WA, Nochimsom DJ, Connolly ME, Fenter MM, Hoehn GJ. The fetal biophysical profile in patients with premature rupture of the membranes. An early predictor of fetal infection. Am J Obstet Gynecol 1985;152:510-6.

20. Garry D. Figueroa R. Aguero-Rosenfeld M, Martínez E, Visintainer P, Tejani N. A comparison of rapid amniotic fluid markers in the prediction of microbial invasion of the uterine cavity and preterm delivery. Am J Obst Gynecol 1996;175:1236-43.

21. Korbage de Araujo MC, Schultz R, do Rosario Dias de Oliveira L, Ramos JL, Vaz FA. A risk factor for earlyonset infection in premature newborns: invasion of chorioamniotic tissues by leukocytes. Early Hum Dev 1999;56:1-15.

22. Yoon BH, Romero R, Moon JB, Oh SY, Han SY, Kim $\mathrm{JC}$, et al. The frequency and clinical significance of intra-amniotic inflammation in patients with a positive cervical fetal fibronectin. Am J Obstet Gynecol 2001;185:1137-42.

23. Rizzo G, Capponi A, Rinaldo D, Tedeschi D, Arduini D, Romanini C. Interleukin-6 concentrations in cervical secretions identify microbial invasion of the amniotic cavity in patients with preterm labor and intact membranes. Am J Obstet Gynecol 1996;175:812-7.
24. Mozurkewich EL, Wolf FM. Premature rupture of membranes at term: a meta-analyses of three management schemes. Obstet Gynecol 1997;89:1035-43.

25. Gibbs RS, Dinsmoor MJ, Newton ER, Ramamurthy RS. A randomized trial of intrapartum versus immediate postpartum treatment of women with intra-amniotic infection. Obstet Gynecol 1988;72:823-8.

26. Kenyon S, Boulvain M, Neilson J. Antibiotics for preterm rupture of membranes. The Cochrane Library, volume 1; 2003.

27. Gibbs RS. Intraamniotic infection. En: Sweet RL, Gibbs RS, editors. Infectious diseases of the female genital tract, 4th ed. Philadelphia, PA: Lippincott Williams \& Wilkins; 2002. p. 520.

28. Gilstrap LC 3rd, Cox SM. Acute chorioamnionitis. Obstet Gynecol Clin North Am 1989;16:373-9.

29. Duff P. Antibiotic selection for infections in obstetric patients. Semin Perinatol 1993;17:367-78.

30. Prevedourakis CN, Strigou-Charabalis E, Kaskarelis DB. Bacterial invasion of amniotic cavity during pregnancy and labor. Obstet Gynecol 1971;37:459-61.

31. Cifuentes R. Obstetricia de alto riesgo. Cali: Aspro médica; 1994. p. 461-76.

32. Gibbs RS. Subclinical infection and premature birth. Abstracts of "The world symposium of perinatal medicine". Washington, USA, 1995.

33. Ballard PL, Ballard RA. Scientific basis and therapeutic regimens for use of antenatal glucocorticoids. Am J Obstet Gynecol 1995;173:254-62.

34. McDonald HM, O 'Loughlin JA, Jolley P, Vigneswaran R, McDonald PJ. Prenatal microbiological risk factors associated with preterm birth. Br J Obstet Gynecol 1992;99:90-6.

35. Goldenberg R, Hauth J, Andrews W. Intrauterine infection and preterm delivery. $\mathrm{N}$ Engl J Med 2000;342:1500-7.

36. Romero R, Sibai B, Caritis S, Paul R, Depp R, Rosen $\mathrm{M}$, et al. Antibiotic treatment of preterm labor with intact membranes. A multicenter, randomized, doubleblinded, placebo-controlled trial. Am J Obstet Gynecol 1993;169:764-74.

37. Mitra AG, Whitten K, Laurent SL, Anderson WE. A randomized, prospective study comparing oncedaily gentamicin versus thrice-daily gentamicin in the treatment of puerperal infection. Am J Obstet Gynecol 1997;177:786-92. 
38. Sweet RL, Gibbs RS. Antimicrobial agents. En: Sweet RL, Gibbs RS. Atlas of infectious diseases of the female genital tract. 4th ed. Philadelphia (PA): Lippincott Williams \& Wilkins; 2002. p. 609-60.

39. Gilstrap LC 3rd, Leveno KJ, Cox SM, Burris JS, Mashbum M, Rosenfeld CR. Intrapartum treatment of acute chorioamnionitis: Impact of neonatal sepsis. Am J Obstet Gynecol 1988;159:579-83.
40. Mazor M, Chaim W, Pack I, Goldstein D. Intraamniotic infection with Candida albicans associated with a retained intrauterine device. A case report. J Reprod Med 1992;37:950-2.

41. Segal D, Gohar J, Huieihel M, Mazor M. Fetal death associated with asymptomatic intrauterine Candida albicans infection and retained intrauterine contraceptive device. Scand J Infect Dis 2001;33:77-8. 